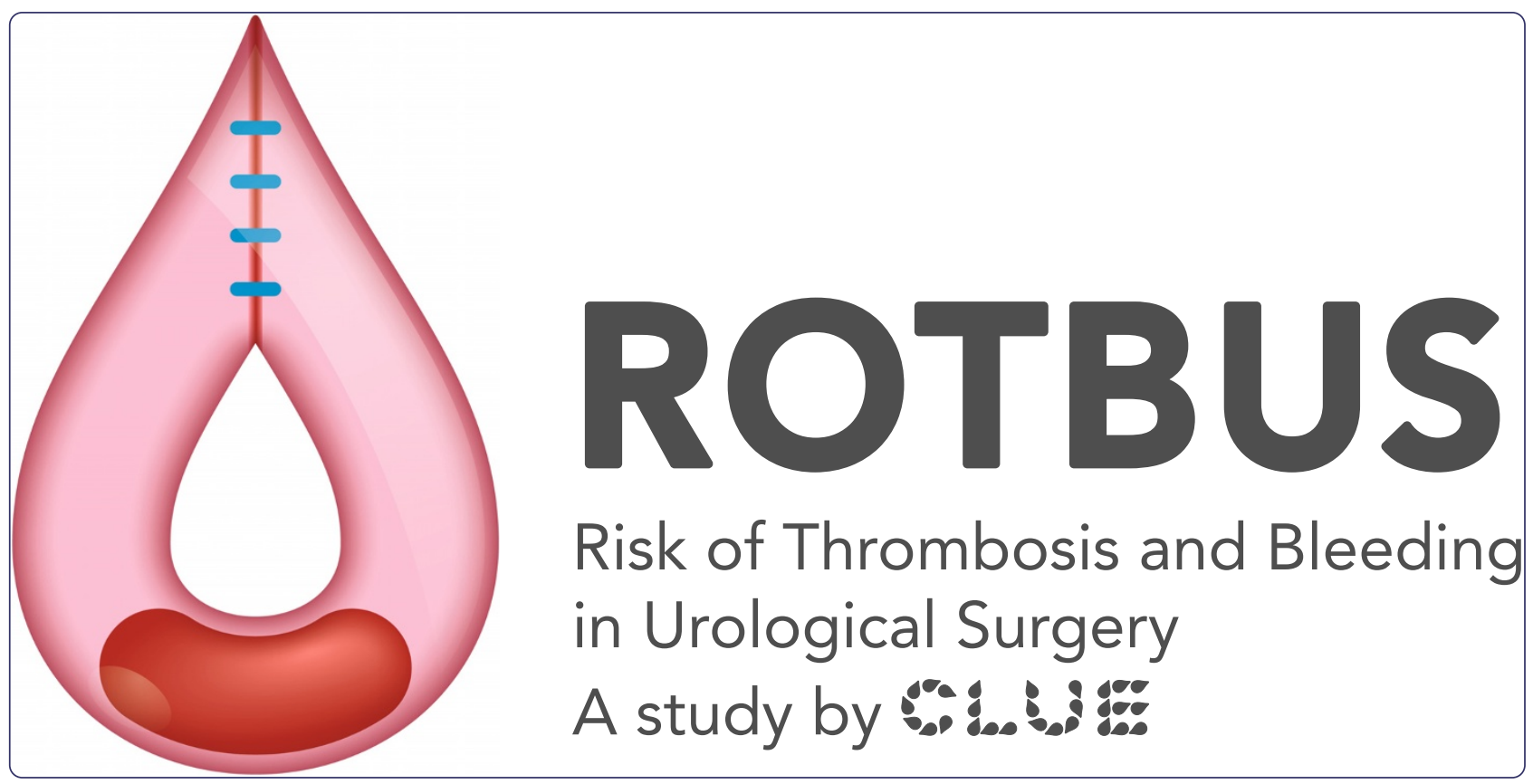

Systematic reviews of observational studies of risk of thrombosis and bleeding in urological surgery (ROTBUS): introduction and methodology

Tikkinen et al. 


\title{
Systematic reviews of observational studies of risk of thrombosis and bleeding in urological surgery (ROTBUS): introduction and methodology
}

Kari AO Tikkinen ${ }^{1,2^{*}}$, Arnav Agarwal ${ }^{3,4}$, Samantha Craigie ${ }^{3,15}$, Rufus Cartwright ${ }^{5,6}$, Michael K Gould ${ }^{7}$, Jari Haukka ${ }^{2}$, Richard Naspro ${ }^{8}$, Giacomo Novara ${ }^{9}$, Per Morten Sandset ${ }^{10,11}$, Reed A Siemieniuk ${ }^{12}$, Philippe D Violette $^{13}$ and Gordon H Guyatt 3,14

\begin{abstract}
Background: Pharmacological thromboprophylaxis in the peri-operative period involves a trade-off between reduction in venous thromboembolism (VTE) and an increase in bleeding. Baseline risks, in the absence of prophylaxis, for VTE and bleeding are known to vary widely between urological procedures, but their magnitude is highly uncertain. Systematic reviews and meta-analyses addressing baseline risks are uncommon, needed, and require methodological innovation. In this article, we describe the rationale and methods for a series of systematic reviews of the risks of symptomatic VTE and bleeding requiring reoperation in urological surgery.

Methods/design: We searched MEDLINE from January 1, 2000 until April 10, 2014 for observational studies reporting on symptomatic VTE or bleeding after urological procedures. Additional studies known to experts and studies cited in relevant review articles were added. Teams of two reviewers, independently assessed articles for eligibility, evaluated risk of bias, and abstracted data. We derived best estimates of risk from the median estimates among studies rated at the lowest risk of bias. The primary endpoints were 30-day post-operative risk estimates of symptomatic VTE and bleeding requiring reoperation, stratified by procedure and patient risk factors.

Discussion: This series of systematic reviews will inform clinicians and patients regarding the trade-off between VTE prevention and bleeding. Our work advances standards in systematic reviews of surgical complications, including assessment of risk of bias, criteria for arriving at best estimates of risk (including modeling of timing of events and dealing with suboptimal data reporting), dealing with subgroups at higher and lower risk of bias, and use of the Grading of Recommendations Assessment, Development and Evaluation (GRADE) approach to rate certainty in estimates of risk. The results will be incorporated in the upcoming European Association Urology Guideline on Thromboprophylaxis.
\end{abstract}

Systematic review registration: PROSPERO CRD42014010342.

Keywords: Baseline risk, Bleeding, Hemorrhage, Modeling, Risk of bias, Surgical complications, Thromboprophylaxis, Thrombosis, Urology

\footnotetext{
* Correspondence: kari.tikkinen@gmail.com

'Department of Urology, Helsinki University Central Hospital and University of Helsinki, Helsinki, Finland

2Department of Public Health, University of Helsinki, Helsinki, Finland

Full list of author information is available at the end of the article
} 


\section{Background Introduction}

Venous thromboembolism (VTE), which includes deep vein thrombosis (DVT) and pulmonary embolism $(\mathrm{PE})$, represents a serious, and sometimes fatal, complication of urological surgery. A systematic review and meta-analysis including gastrointestinal, urological, gynecological, and general surgical procedures has demonstrated that pharmacological prophylaxis decreases the risk of VTE in surgical patients by approximately $50 \%$ but increases the risk of post-operative major bleeding by approximately $50 \%$ [1]. The decision to use pharmacological prophylaxis therefore presents a tradeoff between a reduction in VTE and an increase in bleeding.

The crucial issue in making this decision is the risk of VTE and bleeding in those not receiving anticoagulants, which we will refer to as 'baseline risk'. Baseline risks, in the absence of prophylaxis, for VTE and bleeding are known to vary widely between urological procedures [2], but their magnitude is highly uncertain. In patients with a high risk of VTE and a low risk of bleeding, a $50 \%$ reduction in VTE represents substantial benefit (for instance, from a baseline risk of $10 \%$ to $5 \%$ ). In a patient with a low baseline risk of bleeding (for instance, from $0.2 \%$ to $0.3 \%$ ), the balance of benefits and harms clearly favors prophylaxis. The situation is reversed in patients whose risk of VTE without anticoagulation is low and whose bleeding risk is high. A small reduction in VTE will not warrant the substantial increase in bleeding. In patients whose risk of VTE and bleeding are similar, the decision will depend on the relative value patients place on avoiding VTE and avoiding bleeding.

Thus, the decision regarding thromboprophylaxis is critically dependent on the baseline risks of both VTE and bleeding. Reliable estimates of these risks require systematic summaries of the best available evidence, which have thus far been unavailable [3]. Consequently, the approach to thromboprophylaxis related to urological surgery is an area with marked practice variation, both within and between countries $[4,5]$.

Our series of systematic reviews aims to fill this critical gap in knowledge by addressing the risks of VTE and bleeding requiring reoperation in patients undergoing urological surgery but not receiving prophylaxis. Each review summarizes the evidence regarding the frequency of VTE and bleeding requiring reoperation for procedures for diseases of the urinary tract and (male) genital system. In this article, we outline the specific methods used in our reviews and familiarize readers with the methodology of systematic reviews and metaanalysis of observational studies of complications and their strengths and limitations.
What are systematic reviews of baseline risk, and why are they important but challenging to perform?

Systematic reviews are summaries of a body of evidence, and meta-analysis is a quantitative, statistical method to summarize the results. Preparing a systematic review requires a number of decisions including determining the focus; identifying, selecting, and critically appraising the relevant primary studies; collecting and synthesizing the relevant information; and drawing conclusions from the evidence. Typically, investigators addressing questions of therapy frame their research questions in accord with the Patient, Intervention, Comparator and Outcome (PICO) format with associated eligibility criteria [6]. This facilitates the formulation of an answerable question from which estimates of effect can be derived.

Conventional systematic reviews that compare one treatment against another or against a non-treatment control are common and the methods are well established [6]. Systematic reviews and meta-analyses addressing baseline risks (that is, risk in the absence of intervention, in this case antithrombotic prophylaxis-of important adverse outcomes, in this case VTE or bleeding requiring reoperation) are sorely needed and require methodological innovation [7]. The rates of events in the absence of prophylaxis, required for estimation of benefits and risks, can come only from systematic reviews of the relevant studies. Nevertheless, few such systematic reviews have been undertaken in any area of surgery [8] and none in urology [3].

One reason for the dearth of such reviews is the multiple challenges they present. First, indexing of observational research is less well established than for randomized controlled trials (RCTs), making it harder to identify the relevant studies [9]. Second, surgical studies often focus on disease-related outcomes (for instance, radical prostatectomy papers usually report prostate cancerspecific outcomes) but typically not on generic complications (including VTE and bleeding requiring reoperation).

Third, even when articles report risks of VTE and bleeding, key information is often missing. Such information includes the use of prophylaxis (usually varying among patients), the timing of the events of interest, and diagnostic criteria for the events of interest. Fourth, risk of bias criteria, as well as criteria for overall certainty in estimates [10], well established for reviews of therapeutic trials are controversial in studies of baseline risk [7]. Fifth, the choice of studies to use for best estimates (e.g., synthesis from all studies or only from those at lowest risk of bias) is uncertain [10]. Sixth, the incidence of VTE has changed over time due to advances in surgical technique and care (e.g., early mobilization) [11-13].

In these reviews, we address these challenges with the goal of providing precedents for optimal methodology. For the overall certainty in estimates of effect, we used 
the Grading of Recommendations Assessment, Development and Evaluation (GRADE) system, which has provided detailed methodological guidance for systematic reviews [14], and initial standards for the assessment of baseline risk [7].

\section{Methods/design \\ Eligibility criteria}

We included studies published in English language medical journals that enrolled adult male or female patients undergoing procedures for diseases of the urinary tract and (male) genital system, including kidneys, ureters, bladder, prostate, seminal vesicles, urethra, scrotum, testicles, penis, and vagina (Additional files 1 and 2). Reasoning that very small studies are likely to be published only if they show anomalous results, we included only studies with at least 50 adult patients per urological procedure to decrease risk of bias.

Although RCTs provide estimates of effect of treatment with the lowest risk of bias, populations enrolled are usually highly selected and RCTs are therefore, with respect to estimating baseline risk, limited in terms of generalizability $[15,16]$. Hence, observational studies of unselected patients undergoing urological surgery are likely to be the best source of estimates of VTE and bleeding risk. Because baseline risk has changed over time [11-13], we included studies that recruited all or a majority of participants after the year 2000. Because the complication estimates depend on the length of followup, we included only studies that clearly defined the time period of follow-up (up to 3 months).

Finally, we included only studies that reported at least one absolute estimate of risk of the patient important outcomes of interest (fatal PE, symptomatic PE, symptomatic DVT, symptomatic VTE, fatal bleeding, and bleeding requiring reoperation). Absolute estimates include the percentage (e.g., 1.0\%), proportions (e.g., 0.02), natural units or natural frequency (e.g., 3 in 1,000 patients), and natural frequency per time (e.g., 3 in 1,000 patient years) but do not include relative estimates, such as risk ratio or odds ratio.

Literature search, study selection, application of eligibility criteria, and data abstraction

We used two search strategies. First, we used the MEDLINE database to search for potentially eligible articles published from January 1, 2000 until April 10, 2014. A combination of keyword and medical subject headings search included the 'urological procedures' (more than 90 different urological procedures) term family combined with the 'thrombosis' term family (Additional file 1) as well as the 'urological procedures' term family combined with the 'bleeding' term family and the prognosis sensitivity filter (Additional file 2). We included articles not in the search but known to the experts in the panel. Finally, we identified further original articles by reviewing the reference lists of included systematic reviews (none of the systematic reviews summarized any of the outcomes of interest of our series).

Two reviewers evaluated titles and abstracts from the search and identified potentially eligible studies. We obtained the full articles of these potentially eligible titles and abstracts, and two reviewers assessed the full texts to make final judgments regarding eligibility. Similarly, two reviewers independently abstracted data including outcomes, study characteristics, and risk of bias. A clinician-methodologist adjudicator resolved disagreements regarding eligibility or study characteristics. Finally, we sent our consensus data extraction to the original authors of each article for confirmation or correction. When needed, we also asked authors to clarify details regarding thromboprophylaxis, surgical technique (such as pelvic lymph node dissection), as well as other missing or unclear information.

\section{Outcomes}

Primary outcomes were the absolute risks of symptomatic VTE and bleeding requiring reoperation (including exploration and angioembolization). Secondary outcomes were the absolute risks of fatal PE and fatal bleeding. All outcomes were extracted and analyzed separately for each procedure (see 'Analysis' section).

We also extracted the length of follow-up regarding VTE and bleeding outcomes. When authors presented the frequency of events at more than one time point, we recorded the number of events up to 3 months and used the absolute risk closest to 4 weeks (our primary outcome). We did not collect data on events of questionable importance to patients [17], in particular asymptomatic DVT, changes in hemoglobin levels, or amount of estimated blood loss during the operation. Transfusion rates were not recorded primarily due to expected large differences in clinical practice that would make it difficult to estimate the trade-off of thrombosis and bleeding.

\section{Final selection of eligible studies (risk of bias and outliers)} We assessed design features that could potentially bias the estimates of VTE or bleeding risk. These include the representativeness of the recruited patient population, study type, losses to follow-up, explicitness of criteria for VTE diagnosis, thromboprophylaxis documentation, and data source (Table 1). We also collected information on several other characteristics of the articles and their study populations that may be predictive of VTE or bleeding requiring reoperation (Table 2).

To assess applicability and representativeness of each study and to assess heterogeneity between estimates, we recorded the mean age of the study population and 
Table 1 Design features considered for assessment of risk of bias

\begin{tabular}{lll}
\hline Domain & Lower risk of bias & Higher risk of bias \\
\hline $\begin{array}{l}\text { Sampling and representativeness } \\
\text { of the population }\end{array}$ & $\begin{array}{l}\text { Consecutive patient recruitment or administrative } \\
\text { database with random sampling }\end{array}$ & $\begin{array}{l}\text { Non-consecutive patient recruitment or administrative } \\
\text { database with non-random sampling } \\
\text { Study type }\end{array}$ \\
$\begin{array}{lll}\text { International multicenter; multicenter in one } \\
\text { country; single center, not single surgeon }\end{array}$ & Single surgeon series \\
Source of information & Data abstracted by investigators from patient charts & Administrative database information \\
Thromboprophylaxis documentation & Reporting of patients thromboprophylaxis & No reporting of patients' thromboprophylaxis \\
Diagnostic criteria & $\begin{array}{l}\text { Objective confirmation of symptomatic } \\
\text { venous thromboembolism }\end{array}$ & No objective confirmation of symptomatic \\
Loss to follow-up & Less than 20\% loss to follow-up & 20\% or more loss to follow-up
\end{tabular}

proportion of patients with malignant disease. When there were clear outliers, with atypical populations for either of these factors, we excluded those studies.

\section{Analysis \\ Choosing best estimate}

We used the median value of estimates from studies with the lowest risk of bias to estimate baseline risk of VTE and bleeding requiring reoperation. The reason we chose the median as opposed to the pooled estimates across studies is that even the largest studies are likely to have factors idiosyncratic to that population and setting that will influence risks of both thrombosis and bleeding. There is little reason, given these idiosyncratic factors, that larger studies should have more weight than smaller studies. Under these circumstances, the median

Table 2 Characteristics assessed

\begin{tabular}{|c|c|}
\hline \multicolumn{2}{|l|}{ Characteristics } \\
\hline Year of publication & Patient recruitment (first and last year) \\
\hline Source of sampling a & Study type ${ }^{d}$ \\
\hline Country/countries & Multinational (yes/no) \\
\hline Urological procedure(s) & Total number of patients \\
\hline Gender distribution & Age (mean/median/threshold) \\
\hline $\begin{array}{l}\text { Proportion of patients } \\
\text { with malignant disease }\end{array}$ & $\begin{array}{l}\text { Use and extension of pelvic } \\
\text { lymph node dissection }\end{array}$ \\
\hline $\begin{array}{l}\text { Patient use of mechanical } \\
\text { thromboprophylaxis }\end{array}$ & Patient use of anticoagulants ${ }^{\mathrm{e}}$ \\
\hline $\begin{array}{l}\text { Patient use of aspirin or } \\
\text { other antiplatelet drugs }{ }^{c}\end{array}$ & $\begin{array}{l}\text { Patient use of both mechanical and aspirin/ } \\
\text { anticoagulants } s^{b, c, e}\end{array}$ \\
\hline \multicolumn{2}{|c|}{$\begin{array}{l}\text { aEither retrospective case series, register/administrative database, or } \\
\text { prospective cohort study. } \\
\text { bIncluding antithrombosis stockings, intermittent pneumatic compression } \\
\text { devices, and foot-pumps. } \\
\text { Including aspirin, clopidogrel, dipyridamole, prasugrel, ticagrelor, ticlopidine, } \\
\text { cilostazol, abciximab, eptifibatide, tirofiban, as well as thromboxane inhibitors, } \\
\text { thromboxane synthase inhibitors, thromboxane receptor antagonists, } \\
\text { and terutroban. } \\
{ }^{d} \text { Either single-surgeon series; single center, not single surgeon; multicenter in } \\
\text { one country; international multicenter. } \\
\text { e Including warfarin, low molecular weight heparin, low dose unfractioned } \\
\text { heparin, rivaroxaban, dabigatran, apixaban, fondaparinux, and idraparinux. }\end{array}$} \\
\hline
\end{tabular}

is likely to provide better estimates of typical risk than is the pooled estimate [18].

\section{Modeling risk of VTE over time}

We provided procedure-stratified estimates for both risk of VTE and bleeding requiring reoperation in urological surgery. We chose one and four-week time frames for estimates of risks of thrombosis and bleeding because these are feasible and frequently chosen time frames for, respectively, shorter and longer term prophylaxis. For studies that did not report VTE estimates using these intervals, we modeled estimates-based on a literature search (Additional file 3)-using large-scale populationbased studies (Amin, Beral, and Sweetland, personal communications) $[19,20]$ that have provided data regarding the timing of post-surgical VTE. These results are consistent with recent report using nationwide cystectomy data from the United States [21]. To calculate absolute risk of VTE by post-operative day, we calculated the mean values (of VTE risk) from the available studies $[19,20]$. After assessing mean values for both studies and when calculating the final model for VTE (Figures 1 and 2), interpolated values were calculated using natural cubic spline interpolation [22] and $\mathrm{R}$ data analysis language [23].

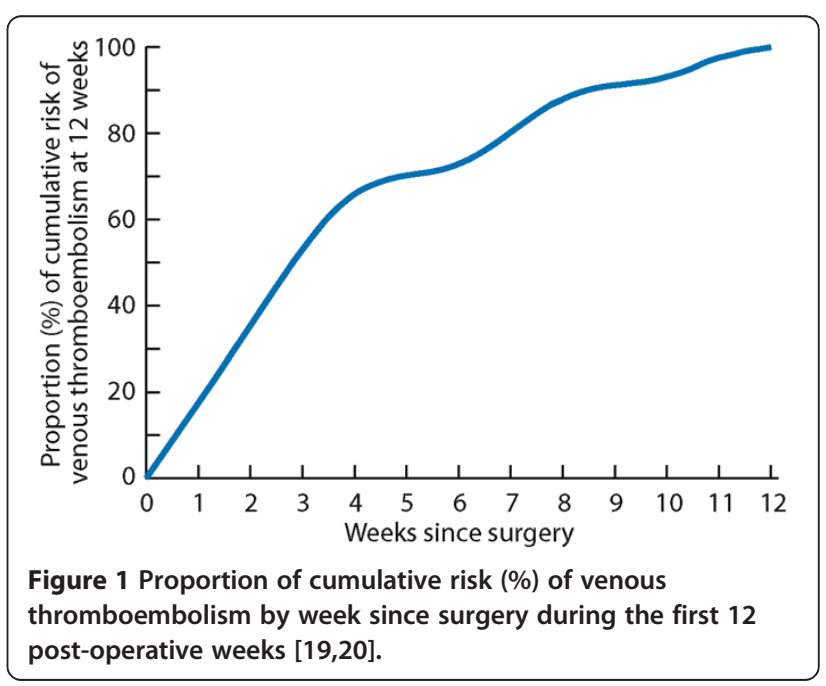




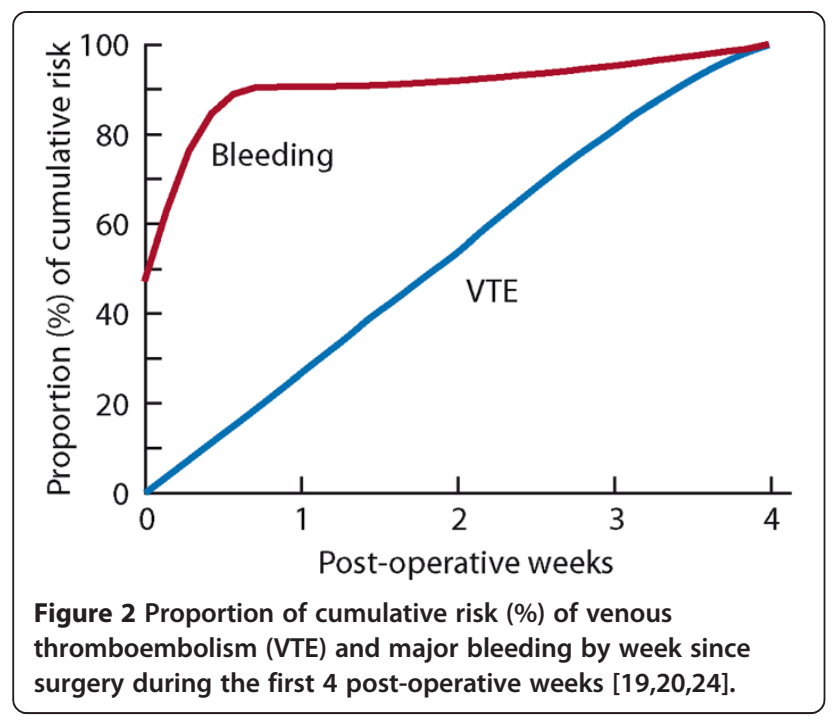

When creating the model for timing of VTE (Figure 1), we used the thromboprophylaxis estimates from the population-based US study [17] where $81.4 \%$ had used either mechanical or pharmacological prophylaxis until discharge (i.e., $81.4 \%$ used for median of 4.5 days) and that $1.5 \%$ used from discharge (median discharge time 4.5 days) until median time of 35 days.

\section{Modeling risk of bleeding over time}

For studies that did not report their bleeding estimates at 4 weeks, we modeled timing of bleeding using data from the placebo arm of a large pragmatic RCT [24]. Ninety percent of the 30-day bleeding events happen during the first week after surgery (and indeed, approximately $75 \%$ in the first 2 days), so that only a small proportion of bleeding happens after the first post-operative week (Figure 2). For studies that provided bleeding data for a longer period than 30 days (but not more than 90 days), we therefore assumed a constant risk of bleeding beyond the first post-operative week, so for example, the day 30 rates were calculated at $80 \%$ of the rates at day 90 (Figure 2).

\section{Calculating risks}

We extracted information from contemporary observational studies. However, one of the challenges of these reviews relates to the decreasing incidence of VTE as surgical techniques have improved and early mobilization has become a standard of care [11-13]. Furthermore, in the recent studies representing current rates of VTE, patients often received prophylaxis. How then to estimate baseline risk?

To adjust estimates of baseline risk for use of prophylaxis, we used information from RCTs and meta-analyses of RCTs about the relative risk of VTE and bleeding among those who received prophylaxis [1,24-26]. Specifically, we used estimates from a meta-analysis of RCTs in urology, general surgery, gynecology, and gastrointestinal surgery that concluded that anticoagulants (such as low molecular weight heparin, see Table 2) reduce the relative risk of VTE by $50 \%$ [1] and increases the relative risk of major bleeding by $50 \%$. We used data from a meta-analysis of RCTs in orthopedic surgery [26] for our estimate that direct oral anticoagulants (Table 2) had similar effects on both VTE and bleeding as low molecular weight heparin. Based on a meta-analysis of RCTs in urology, general surgery, gynecology, and gastrointestinal surgery, we estimated the same efficacy of 50\% VTE risk reduction for mechanical prophylaxis (Table 2) [1]. However, we had low certainty in the estimates for mechanical prophylaxis because studies used surrogate outcomes, had very few events, unblinded patients and assessors, and provided almost no information on intermittent pneumatic compression. Hence, we did not consider combination therapy as offering more protection than heparin alone when adjusting for prophylaxis in the baseline risk estimates of VTE. Finally, we assumed that aspirin (and other antiplatelet drugs, see Table 2) reduces the relative risk of VTE by $30 \%$ and increases the relative risk of major bleeding by $20 \%$, based on two large pragmatic surgical trials $[24,25]$.

We then adjusted the reported risk by multiplying the relative risk by the reported risk in the fraction of patients who received prophylaxis. For instance, a study in which all patients received anticoagulant prophylaxis for 4 weeks or 28 days (time point of our primary outcome) showed a VTE risk of 1\%, assuming that the relative risk reduction with prophylaxis is approximately $50 \%$, we inferred that the included patients would have experienced a $2 \%$ risk of VTE had they not received prophylaxis.

Similarly, in the same study, the bleeding risk was 3\%, because anticoagulant prophylaxis increases the risk of bleeding by approximately $50 \%$, we inferred a bleeding risk of $2 \%$ without prophylaxis. Thromboprophylaxis was often used for less than 4 weeks. In those circumstances, we estimated its impact using same rationale but also considered the duration of thromboprophylaxis by using our models (Figure 2).

If the study did not provide estimates of VTE but only DVT and/or PE, we calculated the risk of VTE using the following approach. We reviewed data from studies that reported DVT, PE, and VTE totals and estimated the overlap (i.e., patients with both DVT and PE) from these studies. We then applied the degree of overlap to estimate VTE frequency in trials that provided only separate reports of DVT and/or PE.

\section{Stratifying the risk of VTE according to patient risk factors}

After assessing the baseline risk of VTE for each procedure, we stratified the risk by patient risk factors. We conducted a literature search addressing VTE risk factors in the 
context of urology, general surgery, gynecology, and gastrointestinal surgery (Additional file 4). We developed a very simple model for VTE risk based on the studies reporting the most relevant and compelling evidence [27-35]. Risk factors included 1) age more than 75 years, 2) obesity (body mass index of 35 or more), and 3) VTE in a first degree relative (parents, full siblings, or children) -all of these increase the risk approximately twofold. The most important risk factor was prior VTE, with risk ratio of approximately 4. We assumed that patients with any combination of two or more risk factors had a risk ratio of 4 . Using these risk factors, we then categorized risk of VTE as low, medium (risk ratio of 2), and high risk (risk ratio of 4).

To calculate estimates of absolute risks for these groups, for each procedure, we estimated the proportion of patients having each of the risk factors using eligible studies. For both age and body mass index, we used the median value and median SD for estimating risk and assumed a normal distribution. We calculated the proportion of those with history of VTE based on large population-based study [35].

Our search did not reveal studies demonstrating convincing and replicable risk factors for bleeding (Additional file 5). Therefore, we did not stratify bleeding risk by patient specific factors.

\section{Case fatality}

We also estimated case fatality rates for VTE and bleeding requiring reoperation. For case fatality of VTE, we divided the number of fatal PE by the number of symptomatic VTE using studies that provided both estimates. Correspondingly, for case fatality of bleeding requiring reoperation, we divided the number of fatal bleeding by the number of bleeding requiring reoperation.

\section{Discussion}

Our methods meet the criteria for rigorous systematic reviews. We specified explicit eligibility criteria, conducted comprehensive searches (not only for baseline risk but also for timing of complications and patient risk factors), and assessed risk of bias using criteria specific to this review. In addition, teams of two reviewers independently assessed eligibility and risk of bias and extracted data, a third reviewer adjudicated discrepancies, and we sent our consensus of data extraction to the original authors of each article for confirmation or correction. We considered, for the first time in urology, risk of VTE and bleeding separately for each procedure including surgical technique (for instance, open vs. robotic and radical vs. partial). We took into account length of follow-up, use of thromboprophylaxis, and patient risk factors.

Our reviews have limitations. Our analyses reflect published data and we cannot completely exclude publication bias. Because reporting of surgical complications is sporadic and ad hoc, it is possible that literature is biased towards underreporting of events. This is especially true regarding single-surgeon series: individual surgeons experiencing high rates of complications are unlikely to publish their results. Secondly, due to poor reporting standards (both indexing and abstract information), our search likely missed some relevant studies.

We have carefully addressed the many challenges involved in generating best estimates of risk of VTE and bleeding in the absence of antithrombotic prophylaxis in patients undergoing urological surgery. Clinicians can therefore look to the systematic reviews in this series as providing the best current estimates of the risk of both symptomatic VTE and bleeding requiring reoperation in urological procedures. Guideline panels will find these estimates helpful when formulating recommendations for VTE prophylaxis in urological surgery. Moreover, these reviews provide guidance for best practices in systematic reviews of observational data-particularly with regard to surgical complications.

\section{Additional files}

\section{Additional file 1: Search history for baseline risk of VTE. \\ Additional file 2: Search history for baseline risk of major bleeding/ bleeding requiring reoperation.}

Additional file 3: Search history for modeling of risk for venous thromboembolism after surgery.

Additional file 4: Search history for patient related risk factors of venous thromboembolism after surgery.

Additional file 5: Search history for patient related risk factors of major bleeding/bleeding requiring reoperation after surgery.

\section{Abbreviations}

CLUE: clinical urology and epidemiology; DVT: deep vein thrombosis; EAU: European Association of Urology; GRADE: grading of recommendations assessment, development and evaluation; PICO: patient, intervention, comparator and outcome; PE: pulmonary embolism; RCT: randomized controlled trial; ROTBUS: risk of thrombosis and bleeding in urological surgery; VTE: venous thromboembolism.

\section{Competing interests}

KAOT, AA, SC, RC, MKG, JH, PMS, RS, PDV and GHG have no financial conflicts of interest. RN has been an advisory board member for Ipsen and a speaker for Ipsen, Lumenis and Storz. GN has been an advisory board member and speaker for Astellas, GlaxoSmithKline, Lilly, Menarini, Nycomed, Pfizer, Pierre Fabre, and Recordati. KAOT is chairman and RC, MKG, RN, GN, PMS and GHG are executive panel members of the European Association of Urology (EAU) ad hoc guideline panel on Thromboprophylaxis. KAOT is member and GHG is co-chair of the Grading of Recommendations Assessment, Development and Evaluation (GRADE) working group. PMS is chairman of the Norwegian working group on Antithrombotic Therapy.

\section{Authors' contributions}

KAOT conceived the study, developed the initial study design proposal, wrote the first draft of the manuscript, and integrated other authors' comments in the manuscript. GHG conceived the study, developed the initial study design proposal, and critically revised the final manuscript. AA, SC, RC, MKG, JH, RN, GN, PMS, RAS, and PDV provided feedback on the study design, and critically revised the final manuscript. All authors read and approved the final manuscript. 


\section{Acknowledgements}

We would like to thank the Academy of Finland, EAU Guideline Office, Finnish Cultural Foundation, Finnish Medical Foundation, Jane and Aatos Erkko Foundation, and Sigrid Jusélius Foundation for providing grant support for the Clinical Urology and Epidemiology (CLUE) Working Group. We would like to thank Alpesh N. Amin, Valerie Beral, John Eikelboom, Jack Hirsch, and Sian Sweetland for insightful comments regarding data analysis and information scientists Laura Banfield and Neera Bhatnagar for advice regarding literature search strategies. We would also like to thank the Risk of Thrombosis and Bleeding in Urological Surgery (ROTBUS) Working Group members for their contributions to the planning and conducting of this project. ROTBUS Working Group members are Arnav Agarwal, Chika Agbassi, Bassel Ali, Rufus Cartwright, Samantha Craigie, Leyla Eryuzlu, Johanna Geraci, Michael K. Gould, Gordon H. Guyatt, Jari Haukka, Maha Imam, Nofisat Ismaila, Denise Kam, Richard Naspro, Giacomo Novara, Per Morten Sandset, Reed Siemieniuk, Kari A.O. Tikkinen (chairman), Philippe D. Violette, Judi Winkup, and Daniel Yoo.

\section{Funding/support and role of the sponsor}

This research was supported by the Academy of Finland (\#276046), EAU Guidelines Office, Finnish Cultural Foundation, Finnish Medical Foundation, Jane and Aatos Erkko Foundation, and Sigrid Jusélius Foundation. RC was supported by a Research Training Fellowship from the UK Medical Research Council. PDV was supported by the Quebec Urological Association, the University of Western Ontario, and the Frank McGill Travelling fellowship. The sponsors had no role in the analysis and interpretation of the data or the manuscript preparation, review, or approval.

\section{Author details}

'Department of Urology, Helsinki University Central Hospital and University of Helsinki, Helsinki, Finland. 'Department of Public Health, University of Helsinki, Helsinki, Finland. ${ }^{3}$ Department of Clinical Epidemiology and Biostatistics, McMaster University, Hamilton, ON, Canada. ${ }^{4}$ School of Medicine, University of Toronto, Toronto, ON, Canada. ${ }^{5}$ Department of Epidemiology and Biostatistics, Imperial College London, London, UK. ${ }^{6}$ Department of Urogynaecology, St. Mary's Hospital, London, UK. Department of Research and Evaluation, Kaiser Permanente Southern California, Pasadena, CA, USA. ${ }^{8}$ Department of Urology, AO Papa Giovanni XXIII, Bergamo, Italy. ${ }^{9}$ Department of Surgical, Oncological, and Gastroenterological Sciences, Urology Clinic, University of Padua, Padua, Italy. ${ }^{10}$ Department of Haematology, Oslo University Hospital, Oslo, Norway. ${ }^{11}$ Institute of Clinical Medicine, University of Oslo, Oslo, Norway. ${ }^{12}$ Department of Medicine, University of Toronto, Toronto, ON, Canada. ${ }^{13}$ Department of Surgery, Division of Urology, the University of Western Ontario, London, ON, Canada. ${ }^{14}$ Department of Medicine, McMaster University, Hamilton, ON, Canada. ${ }^{15}$ Michael G. DeGroote Institute for Pain Research and Care, McMaster University, Hamilton, ON, Canada.

Received: 13 September 2014 Accepted: 10 December 2014 Published: 23 December 2014

\section{References}

1. Gould MK, Garcia DA, Wren SM, Karanicolas PJ, Arcelus JI, Heit JA, Samama CM, American College of Chest Physicians: Prevention of VTE in nonorthopedic surgical patients: antithrombotic therapy and prevention of thrombosis, 9th ed: American College of Chest Physicians EvidenceBased Clinical Practice Guidelines. Chest 2012, 141(Suppl 2):e227S-e277S.

2. Tyson MD, Castle EP, Humphreys MR, Andrews PE: Venous thromboembolism after urological surgery. J Urol 2014, 192:793-797.

3. Forrest JB, Clemens JQ, Finamore $P$, Leveillee $R$, Lippert $M$, Pisters $L$, Touiier K, Whitmore K, American Urological Association: AUA best practice statement for the prevention of deep vein thrombosis in patients undergoing urologic surgery. J Urol 2009, 181:1170-1177.

4. Galvin DJ, Mulvin D, Quinlan DM: Thromboprophylaxis for radical prostatectomy: a comparative analysis of pre-sent practice between the USA, the UK, and Ireland. Prostate 2004, 60:338-342.

5. Soloway MS: Thromboembolism prophylaxis and total prostatectomy: is pharmacologic therapy required? Eur Urol 2008, 53:21-23.

6. Cochrane Handbook for Systematic Reviews of Interventions Version 5.1.0. www.cochrane-handbook.org/.

7. Spencer FA, lorio A, You J, Murad MH, Schunemann HJ, Vandvik PO, Crowther MA, Pottie K, Lang ES, Meerpohl JJ, Falck-Ytter Y, Alonso-Coello P,
Guyatt GH: Uncertainties in baseline risk estimates and confidence in treatment effects. BMJ 2012, 345:e7401.

8. Januel JM, Chen G, Ruffieux C, Quan H, Douketis JD, Crowther MA, Colin C, Ghali WA, Burnand B, IMECCHI Group: Symptomatic in-hospital deep vein thrombosis and pulmonary embolism following hip and knee arthroplasty among patients receiving recommended prophylaxis: a systematic review. JAMA 2012, 307:294-303.

9. Wieland S, Dickersin K: Selective exposure reporting and Medline indexing limited the search sensitivity for observational studies of the adverse effects of oral contraceptives. J Clin Epidemio/ 2005, 58:560-567.

10. Guyatt GH, Oxman AD, Kunz R, Vist GE, Falck-Ytter $Y$, Schunemann HJ, GRADE Working Group: What is "quality of evidence" and why is it important to clinicians? BMJ 2008, 336:995-998.

11. Engel HJ, Tatebe S, Alonzo PB, Mustille RL, Rivera MJ: Physical therapistestablished intensive care unit early mobilization program: quality improvement project for critical care at the University of California San Francisco Medical Center. Phys Ther 2013, 93:975-985.

12. Mont MA, Jacobs JJ, Boggio LN, Bozic KJ, Della Valle CJ, Goodman SB, Lewis CG, Yates AJ Jr, Watters WC 3rd, Turkelson CM, Wies JL, Donnelly P, Patel N, Sluka P, AAOS: Preventing venous thromboembolic disease in patients undergoing elective hip and knee arthroplasty. J Am Acad Orthop Surg 2011, 19:768-776.

13. Morris BA, Benetti M, Marro H, Rosenthal CK: Clinical practice guidelines for early mobilization hours after surgery. Orthop Nurs 2010, 29:290-316.

14. Guyatt GH, Oxman AD, Vist GE, Kunz R, Falck-Ytter Y, Alonso-Coello P, Schünemann HJ, GRADE Working Group: GRADE: an emerging consensus on rating quality of evidence and strength of recommendations. BMJ 2008, 336:924-926.

15. Van Spall HG, Toren A, Kiss A, Fowler RA: Eligibility criteria of randomized controlled trials published in high-impact general medical journals: a systematic sampling review. JAMA 2007, 297:1233-1240.

16. Heit JA: Estimating the incidence of symptomatic postoperative venous thromboembolism: the importance of perspective. JAMA 2012, 307:306-307.

17. Calvert M, Brundage $M$, Jacobsen PB, Schünemann HJ, Efficace F: The CONSORT Patient-Reported Outcome (PRO) extension: implications for clinical trials and practice. Health Qual Life Outcomes 2013, 11:184-190.

18. Guyatt GH, Oxman AD, Santesso N, Helfand M, Vist G, Kunz R, Brozek J, Norris S, Meerpohl J, Djulbegovic B, Alonso-Coello P, Post PN, Busse JW, Glasziou P, Christensen R, Schünemann HJ: GRADE guidelines 12. Preparing summary of findings tables-binary outcomes. J Clin Epidemiol 2013, 66:158-172.

19. Amin AN, Lenhart G, Princic N, Lin J, Thompson S, Johnston S: Retrospective administrative database study of the risk the time period of venous thromboembolism risk during and following hospitalization for major orthopedic or abdominal surgery in real-world US patients. Hosp Pract 2011, 39:7-17.

20. Sweetland S, Green J, Liu B, Berrington de González A, Canonico M, Reeves G, Beral V, Million Women Study Collaborators: Duration and magnitude of the postoperative risk of venous thromboembolism in middle aged women: prospective cohort study. BMJ 2009, 339:b4583.

21. VanDlac AA, Cowan NG, Chen Y, Anderson RE, Conlin MJ, La Rochelle JC, Amling $C L$, Koppie TM: Timing, incidence and risk factors for venous thromboembolism in patients undergoing radical cystectomy for malignancy: a case for extended duration pharmacological prophylaxis. J Urol 2014, 191:943-947.

22. Hastie TJ, Tibshirani RJ: Generalized Additive Models. In London: Chapman \& Hall; 1990:335p.

23. R Core Team: R: a language and environment for statistical computing. www.R-project.org/.

24. Devereaux PJ, Mrkobrada M, Sessler DI, Leslie K, Alonso-Coello P, Kurz A Villar JC, Sigamani A, Biccard BM, Meyhoff CS, Parlow JL, Guyatt G, Robinson A, Garg AX, Rodseth RN, Botto F, Lurati Buse G, Xavier D, Chan MT, Tiboni M, Cook D, Kumar PA, Forget P, Malaga G, Fleischmann E, Amir M, Eikelboom J, Mizera $R$, Torres $D$, Wang $C Y$, et al: Aspirin in patients undergoing noncardiac surgery. N Engl J Med 2014, 370:1494-1503.

25. Pulmonary Embolism Prevention (PEP) trial Collaborative Group: Prevention of pulmonary embolism and deep vein thrombosis with low dose aspirin: Pulmonary Embolism Prevention (PEP) trial. Lancet 2000, 355:1295-1302. 
26. Neumann I, Rada G, Claro JC, Carrasco-Labra A, Thorlund K, AkI EA, Bates SM, Guyatt GH: Oral direct Factor Xa inhibitors versus low-molecular-weight heparin to prevent venous thromboembolism in patients undergoing total hip or knee replacement: a systematic review and meta-analysis. Ann Intern Med 2012, 156:710-719.

27. Hansson PO, Welin L, Tibblin G, Eriksson H: Deep vein thrombosis and pulmonary embolism in the general population: 'The Study of Men Born in 1913'. Arch Intern Med 1997, 157:1665-1670.

28. Tosetto A, Frezzato M, Rodeghiero F: Prevalence and risk factors of non-fatal venous thromboembolism in the active population of the VITA Project. J Thromb Haemost 2003, 1:1724-1729.

29. Edmonds MJ, Crichton TJ, Runciman WB, Crichton TJ, Runciman WB, Pradhan M: Evidence-based risk factors for postoperative deep vein thrombosis. ANZ J Surg 2004, 74:1082-1097.

30. Stein PD, Hull RD, Kayali F, Ghali WA, Alshab AK, Olson RE: Venous thromboembolism according to age: the impact of an aging population. Arch Intern Med 2004, 164:2260-2265.

31. Weill-Engerer S, Meaume S, Lahlou A, Piette F, Saint-Jean O, Sachet A, Beinis JY, Gallinari C, Grancher AS, Vincent JP, Naga H, Belmin J, Salvatore R, Kazes M, Pautas E, Boiffin A, Piera JB, Duviquet M, Knafo D, Piau A, Miric D, Jean A,

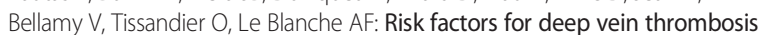
in inpatients aged 65 and older: a case-control multicenter study. J Am Geriatr Soc 2004, 52:1299-1304.

32. Caprini JA: Thrombosis risk assessment as a quide to quality patient care. Dis Mon 2005, 51:70-78.

33. Rogers SO Jr, Kilaru RK, Hosokawa P, Henderson WG, Zinner MJ, Khuri SF: Multivariable predictors of postoperative venous thromboembolic events after general and vascular surgery: results from the patient safety in surgery study. J Am Coll Surg 2007, 204:1211-1221.

34. Parkin L, Sweetland S, Balkwill A, Green J, Reeves G, Beral V, Million Women Study Collaborators: Body mass index, surgery, and risk of venous thromboembolism in middle-aged women: a cohort study. Circulation 2012, 125:1897-1904.

35. Pannucci CJ, Laird S, Dimick JB, Campbell DA, Henke PK: A validated risk model to predict 90-day VTE events in postsurgical patients. Chest 2014 145:567-573.

doi:10.1186/2046-4053-3-150

Cite this article as: Tikkinen et al:: Systematic reviews of observational studies of risk of thrombosis and bleeding in urological surgery (ROTBUS): introduction and methodology. Systematic Reviews 2014 3:150.

\section{Submit your next manuscript to BioMed Central and take full advantage of:}

- Convenient online submission

- Thorough peer review

- No space constraints or color figure charges

- Immediate publication on acceptance

- Inclusion in PubMed, CAS, Scopus and Google Scholar

- Research which is freely available for redistribution 